\title{
1 An analysis of membrane fusion between mitochondrial double membranes and MITO-Porter, 2 mitochondrial fusogenic vesicles
}

4 Yuma Yamada ${ }^{1}$, Yutaka Fukuda ${ }^{1}$, Hideyoshi Harashima ${ }^{1, *}$.

$5 \quad{ }^{1}$ Laboratory for Molecular Design of Pharmaceutics, Faculty of Pharmaceutical Sciences, Hokkaido University, Kita6 12, Nishi-6, Kita-ku, Sapporo 060-0812, Japan

8 *To whom correspondence should be addressed: Laboratory for Molecular Design of Pharmaceutics, Faculty of 9 Pharmaceutical Sciences, Hokkaido University, Kita-12, Nishi-6, Kita-ku, Sapporo 060-0812, Japan. E-mail: harasima@pharm.hokudai.ac.jp. Phone: +81-11-706-3919. Fax: +81-11-706-4879

\section{Keywords:}

Mitochondria; mitochondrial drug delivery; nucleic acid delivery; mitochondrial matrix; MITO-Porter, membrane fusion.

\section{Abstract}

To achieve mitochondrial gene therapy, therapeutic molecules need to be transported through the outer and inner membranes of mitochondria into the innermost space (mitochondrial matrix), which contains the mtDNA pool. We previously reported on the construction of a MITO-Porter with a high fusogenic activity for the mitochondrial outer membrane for delivering molecules to mitochondria of human cells. Here, we report on an investigation of a fusogenic lipid composition for the inner membrane, and an analysis of the fusogenic compositions for the outer and inner membranes. A significant relationship was found between fusion activity and the mitochondrial delivery of nucleic acids. 


\section{Introduction}

It has been reported by numerous investigators that genetic defects in mitochondrial DNA (mtDNA) are associated with mitochondrial diseases and that a variety of human disorders, including neurodegenerative diseases, diabetes mellitus and cancer can be attributed to this (Chan, 2006; Schapira, 2006; Taylor and Turnbull, 2005). Mitochondrial genome-targeting nucleic acids are promising candidates for the therapeutic treatment of mitochondrial diseases. Up to the present, a number of systems for the delivery of nucleic acids to the cytosol and the nucleus, including several successful gene therapies, have been reported (Miyata et al., 2012; Nakamura et al., 2012), while much less progress has been made concerning mitochondrial delivery systems (Biswas and Torchilin, 2014; Kajimoto et al., 2014; Weissig, 2011; Yamada and Harashima, 2008; Zhang et al., 2011). It is noteworthy in this respect that mitochondrial gene therapy has never been achieved. An optimal mitochondrial targeting system for regulating intracellular trafficking and the import therapeutic molecules into the innermost mitochondrial space (the mitochondrial matrix), which contains the mtDNA pool, are required to accomplish mitochondrial gene therapy.

In a previous study, we reported on the development of a Dual Function (DF)-MITO-Porter, an innovative nanocarrier for achieving mitochondrial delivery, which has the ability to pass through the endosomal and mitochondrial membranes via step-wise membrane fusion (Yamada et al., 2011; Yamada and Harashima, 2012). Octaarginine (R8), a cell penetrating peptide, was used to modify the MITO-Porter system, and was found to function as a useful moiety for cellular uptake via macropinocytosis and mitochondrial targeting via electrostatic interaction (Futaki et al., 2001; Khalil et al., 2006; Yamada et al., 2008). In addition, we succeeded in packaging an oligodeoxynucleotide (a model nucleic acid) in the DF-MITO-Porter and were able to achieve the mitochondrial delivery of nucleic acids to regulate the multiple intracellular processes (Yamada et al., 2012e). More recently, the S2 peptide (Dmt- ${ }^{-}$-Arg-FK-Dmt- ${ }^{-}$-Arg-FK- $\mathrm{NH}_{2}$ ) modified DF-MITO-Porter was found to have a lower cell toxicity compared to the R8 modified carrier, while its mitochondrial targeting activity were similar to that of R8 (Kawamura et al., 2013).

Furthermore, we verified that the MITO-Porter delivered cargoes to the mitochondrial matrix using propidium iodide as a probe to detect mtDNA. As result, we were able to confirm that this system can be used to efficiently visualize mtDNA, not only in isolated mitochondria, but in living cells as well (Yasuzaki et al., 2010). We also attempted the mitochondrial delivery of the DNase I protein using the DF-MITO-Porter to estimate the 
mitochondrial gene targeting of the carrier (Yamada et al., 2012a; Yamada et al., 2011; Yamada and Harashima, 2012).

2 The results indicated that the use of the DF-MITO-Porter resulted in a decrease in mtDNA-levels followed by a decrease in mitochondrial activity. Based on these previous reports, we conclude that the MITO-Porter system has the ability to deliver cargoes to the mitochondrial matrix and the target mtDNA.

To efficiently import cargoes to the mitochondrial matrix, the MITO-Porter is required to efficiently fuse with both mitochondrial outer and inner membranes. As previously reported, R8-modified envelopes composed of 1,2-dioleoyl-sn-glycero-3-phosphatidylethanolamine (DOPE) showed a high fusogenic activity with the mitochondrial outer membrane (Yamada et al., 2008), however, these lipids may not be the best lipid composition for use in conjunction with the mitochondrial inner membrane. We report herein on an investigation of a fusogenic lipid composition designed for the mitochondrial inner membrane and a relationship analysis of the lipid composition needed for the mitochondrial outer versus the inner membrane. This analysis for mitochondrial membrane fusion was done by monitoring the cancellation of fluorescence resonance energy transfer (FRET) between donor and acceptor fluorophores, modified on the surface of liposomes (Maier et al., 2002; Struck et al., 1981; Yamada et al., 2012a; Yamada et al., 2008; Yamada and Harashima, 2014). We also evaluated the import of nucleic acids to the mitochondrial matrix by the MITO-Porter system, and investigated the effect of fusion activity for outer and inner membranes on import efficiency.

\section{Materials and methods}

\subsection{Materials}

Cholesterol (Chol), cardiolipin (CL), 1, 2-dioleoyl-sn-glycero-3-phosphatidyl ethanolamine (DOPE), phosphatidyl glycerol (PG), phosphatidyl serine (PS), sphingomyelin (SM), 7-nitrobenz-2-oxa-1, 3-diazole labeled DOPE (NBD-DOPE) and rhodamine-DOPE were purchased from Avanti Polar lipids (Alabaster, AL, USA). Egg yolk phosphatidyl choline (EPC) was obtained from the NOF Corporation (Tokyo, Japan). Cholesteryl hemisuccinate (CHEMS), phosphatidic acid (PA), phosphatidyl inositol (PI) and were obtained from Sigma-Aldrich (St. Louis, MO, USA). Stearylated R8 (STR-R8) was obtained from Kurabo Industries Ltd (Osaka, Japan). Cy5-labeled 2'-OMe RNA oligonucleotide (Cy-5 RNA oligo) (5'- CUUGCGCUGCAUGUGCCAU -3') was purchased from Greiner Bio-One 
1 Supplementary material. All other chemicals used were commercially available reagent-grade products.

\subsection{Experimental animals}

Male Wistar rats (6-8 weeks old) were purchased from Sankyo Labo Service (Sapporo, Japan). Rats with body

weights in the range 140-200 g were used in all experiments. All animal protocols were approved by the institutional animal care and research advisory committee at the Faculty of Pharmaceutical Sciences, Hokkaido University, Sapporo, Japan (date: 22 March 2013, registration no. 13-0062).

\subsection{Preparation of mitoplast suspensions}

The mitoplast, which is mitochondrion with the outer membrane removed, was prepared by digitonin treatment followed by differential centrifugation (see the supplementary material for details) (Greenawalt, 1974; Yamada et al., 2008). The purity of the mitoplast solutions was confirmed by western blotting to detect mitochondrial outer membrane specific proteins, the voltage-dependent anion channel 2 (VDAC2) and mitochondrial matrix specific proteins, the pyruvate dehydrogenase E1-alpha subunit (PDHE1- $\alpha$ ) (see the supplementary material for details).

\subsection{Liposome preparation and packaging of nucleic acids}

To investigate mitochondrial fusogenic activity using isolated mitochondria and a FRET analysis, duallabeled liposomes containing both $1 \mathrm{~mol} \% \mathrm{NBD}-\mathrm{DOPE}$ and $0.5 \mathrm{~mol} \%$ rhodamine-DOPE were prepared with various lipid compositions by a previously reported hydration method (Yamada et al., 2008; Yamada and Harashima, 2014).

The lipid compositions of these liposomes are summarized in Table 1. Lipid films were produced on the bottom of a glass tube by the evaporation of a chloroform/ethanol solution containing $137.5 \mathrm{nmol}$ lipids. After the formation of the film, $250 \mu \mathrm{L}$ of mitochondrial isolation buffer (MIB: $250 \mathrm{mM}$ sucrose, $2 \mathrm{mM}$ Tris- $\mathrm{HCl}$, $\mathrm{pH}$ 7.4) was added, followed by incubation for $15 \mathrm{~min}$ at room temperature and sonication for $30 \mathrm{sec}$ in a bath-type sonicator (85W, Aiwa, Co., Tokyo, Japan). To attach R8 to the surface of the carrier, a solution of STR-R8 (10 mol\% lipids) was added to the resulting suspensions, followed by incubation for $30 \mathrm{~min}$ at room temperature. In this experiment, Cy-5 RNA oligo, a 19 base Cy5-labeled 2'-OMe RNA oligonucleotide, was used as a model cargo nucleic acid to evaluate the 
mitochondrial delivery of the carrier. To encapsulate the Cy-5 RNA oligo in the carrier, a Cy-5 RNA oligo complex with polycation was formed, and then packaged within the lipid envelopes by the hydration method (see the supplementary material for details) (Kogure et al., 2004; Yamada et al., 2012e). Particle diameters were measured using a dynamic light scattering method (Zetasizer Nano ZS; Malvern Instruments, Worcestershire, UK). Samples were measured in MIB at $25^{\circ} \mathrm{C}$ and the values of particle diameters are shown in the form of volume distribution. The $\zeta$-potentials of samples were also determined in MIB at $25^{\circ} \mathrm{C}$ using a Zetasizer Nano ZS.

\subsection{Membrane fusion assay using FRET analysis}

The membrane fusion activity of the liposomes with mitochondria was assessed by FRET between donor and acceptor fluorophores, that were modified on the surface of the liposomes, as previously described (Maier et al., 2002; Struck et al., 1981; Yamada et al., 2012a; Yamada et al., 2008; Yamada and Harashima, 2014). In this experiment, liposomal membranes were labeled with both NBD (excitation at $460 \mathrm{~nm}$ and emission at $534 \mathrm{~nm}$ ) and rhodamine (excitation at $550 \mathrm{~nm}$ and emission at $590 \mathrm{~nm}$ ) so that energy would be transferred from the NBD to rhodamine. Membrane fusion between the dual-labeled liposomes and the mitochondria would lead to the diffusion of NBD and rhodamine into the lipid membranes, which would cause a reduction in energy transfer, resulting in an increase in the fluorescence intensity at $530 \mathrm{~nm}$ (Figure 1A). A 10- $\mu \mathrm{L}$ aliquot of the dual-labeled liposome (lipid concentration, $0.55 \mathrm{mM})$ was added to a mitochondrial suspension or a mitoplast solution $(1 \mathrm{mg}$ of protein $/ \mathrm{mL})$ in $90 \mu \mathrm{L}$ of MIB on a 96 well black plate (Greiner Bio-One GmbH, Frickenhausen, Germany), which was then incubated for $30 \mathrm{~min}$ at $25^{\circ} \mathrm{C}$. After the incubation, the energy transfer was assessed by measuring the fluorescence intensity (excitation at $470 \mathrm{~nm}$ and emission at $530 \mathrm{~nm}$ ) using an EnSpireTM 2300 Multilabel Reader (PerkinElmer, Inc., Waltham, MA). The maximum fluorescence was defined as the fluorescence of liposomes when dissolved in Triton X-100 (final concentration, 0.5\% (v/v)). Fusion activity (\%) was estimated by the reduction in the level of energy transfer in accordance with membrane fusion, and was calculated as follows:

$$
\text { Fusion activity }(\%)=\left(\mathrm{F}-\mathrm{F}_{0}\right) /\left(\mathrm{F}_{\max }-\mathrm{F}_{0}\right) \times 100
$$

where $\mathrm{F}, \mathrm{F}_{0}$ and $\mathrm{F}_{\max }$ represent the fluorescence intensity of labeled liposome after incubation with mitochondria or mitoplast, the fluorescence intensity of labeled liposome after incubation without mitochondria, and the maximum fluorescence intensity after the Triton X-100 treatment, respectively. 
2.6. Evaluation of mitochondrial targeting and the matrix import of nucleic acids

A suspension of isolated mitochondria $270 \mu \mathrm{L}(1 \mathrm{mg}$ protein $/ \mathrm{mL})$ and a Cy-5 RNA oligo encapsulated carrier $30 \mu \mathrm{L}$ (lipid concentration $0.55 \mathrm{mM}$ ) were mixed and the resulting suspension incubated for $30 \mathrm{~min}$ at $25^{\circ} \mathrm{C}$. A portion of the resulting solution was treated with poly aspartic acid (pAsp) and denoted as sample A. The remaining suspension was centrifuged $\left(7,500 \mathrm{~g}, 10 \mathrm{~min}, 4^{\circ} \mathrm{C}\right)$, and washed twice with MIB and re-precipitated by centrifugation $\left(16,000 \mathrm{~g}, 10 \mathrm{~min}, 4^{\circ} \mathrm{C}\right)$. The pellet was resuspended in MIB containing pAsp, and a portion of the suspension was used as sample B. The remaining suspension was mixed with an equal volume of a $0.03 \%$ digitonin solution, and the mitoplast suspension was then prepared (Sample C) as described above. Samples A, B and C were dissolved in sodium dodecyl sulfate (final concentration $0.5 \%$ ) and the fluorescence intensities were measured with excitation at $645 \mathrm{~nm}$ and emission at $660 \mathrm{~nm}$ (FP-6300, JASCO, Tokyo, Japan). Mitochondrial targeting activity and matrix import efficiency were calculated as follows:

\section{Results and discussion}

3.1. Construction of liposomes with various lipid compositions and an evaluation of mitochondrial outer membrane 
fusion activity using isolated mitochondria.

We previously established a method for evaluating the membrane fusion activity of liposomes with isolated rat liver mitochondria and FRET analysis (Yamada et al., 2012a; Yamada et al., 2008; Yamada and Harashima, 2014), as shown in Figure 1A. A previous investigation showed that R8-modified envelopes composed of DOPE showed a high fusogenic activity for the mitochondrial outer membrane (Yamada et al., 2008), moreover, the lipid composition containing SM [DOPE/SM/STR-R8 (9:2:1, molar ratio)] was optimal for use in constructing the MITO-Porter, since it had the highest mitochondrial fusogenic activity and the lowest cytotoxicity (Yamada et al., 2012a; Yamada et al., 2011). It should be noted that the lipid composition of a conventional MITO-Porter may not be the best lipid composition for use in the mitochondrial inner membrane. Thus, we screened a series of lipid compositions of liposomes with fusogenic activity for the mitochondrial inner membrane. These analyses for membrane fusion were achieved by varying the lipid composition of a panel of liposomes, and high throughput screening to improve the previous FRET analysis by using micro plate reader to avoid complexity or consuming large amount of time.

We first evaluated mitochondrial outer membrane fusion activity using this new method, and validated that the new data for fusion activity were consistent with our previous data. For this screening, various types of liposomes with different lipid compositions were prepared, as described in a previous report (Yamada et al., 2008). Table 1 summarizes the characteristics of the liposomes, including lipid composition, diameter and $\zeta$-potentials. The activities of these liposomes for fusion with the mitochondrial outer membrane are summarized in Figure 1B. We performed a two-way ANOVA analysis to compare the effect of two factors, namely, "lipid composition (EPC-LP group, DOPELP group, R8-EPC-LP group and R8-DOPE-LP group)" and "lipid X component (SM, Chol. CHEMS, PG, PA, CL, PI and PS)". As a result, significant differences were found in the case of "lipid composition" ("\#\#"p $<0.001$ ), where R8-modified liposomes (R8-DOPE-LPs and R8-EPC-LPs) showed higher fusogenic activities than R8-unmodified liposomes (DOPE-LPs and EPC-LPs). In the case of R8-modification, the values for the R8-DOPE-LP were significantly higher than those for the R8-EPC-LP. The finding that the R8-DOPE-LPs showed a high mitochondrial fusogenic activity was in agreement with data from our previous report (Yamada et al., 2008). In this study, R8DOPE-LPs containing SM, PA or PI also exhibited the highest fusion activities among the lipids examined. 

membranes.

We investigated a panel of liposomes for fusogenic activity for the mitochondrial inner membrane as shown in Table 1, using high throughput screening based on a FRET analysis and mitoplasts, which are mitochondria with the outer membrane removed, i.e., the inner membrane with the enclosed matrix. The procedure for preparing mitoplasts includes two steps; digitonin treatment of mitochondria to remove the outer membrane and differential centrifugation to purify the mitoplast as shown in Figure 2A. Mitoplasts were prepared using different concentrations of digitonin, and western blotting analysis permitted us to analyze the remaining outer membrane and the intact structure of the inner membrane of the mitoplast. Each mitoplast sample was subjected to western blotting using primary antibodies against the VDAC2, located on the outer membrane, and PDHE1- $\alpha$, locating in the matrix (Figure 2B). The data showed that no band corresponding to VDAC2 was detected at a digitonin concentration of more than $0.9 \%$, while bands corresponding to PDHE1- $\alpha$ were clearly detected. We also observed that the intensity of bands corresponding to PDHE1- $\alpha$ increased with increasing digitonin concentration. This result indicates that mitoplasts contain much higher levels of matrix proteins, including PDHE1- $\alpha$, than isolated mitochondria, as evidenced by the fact that same amounts of total proteins $(2 \mu \mathrm{g}$ of protein) were used in western blotting, because the levels of outer membrane proteins including, VDAC2, were reduced by the digitonin treatment. Thus, mitoplasts prepared using $0.9 \%$ digitonin, where the inner membrane with the enclosed matrix is left intact, were used to evaluate fusogenic activity for the mitochondrial inner membrane

Figure $2 \mathrm{C}$ shows the evaluation of fusion activities of prepared liposomes for mitochondrial inner membrane. In performing a two-way ANOVA analysis, significant differences were detected for "lipid composition" ("\#\#" < 0.001). Among "lipid composition", R8-modified liposomes (R8-DOPE-LPs and R8-EPC-LPs) showed higher fusogenic activities than R8-unmodified liposomes (DOPE-LPs and EPC-LPs). On the other hand, the values for R8DOPE-LP were comparable to those of R8-EPC-LP, different from the fusion activity for the outer membrane. The results suggest that R8-modification strongly enhances the fusion activity of carriers with respect to the mitochondrial inner membrane in the case of carriers containing DOPE or EPC. As it is well known that cardiolipin is present in high concentrations in the mitochondrial inner membrane and possesses two negatively charges per molecule. Thus, 
inner membrane, which contains large amounts of cardiolipin. One possibility is that strong electrostatic interactions between the cationic carrier and the two negative charges on cardiolipin would be expected to allow the carrier to approach mitochondrial inner membrane closer, this enhancing membrane fusion, although the details of mechanism are not well understood.

Moreover, we investigated the relationship between the mitochondrial outer and inner membrane fusion activities to better understand the characteristics of the fusogenic lipid compositions used. Figure S1 shows the relationship fusion activity between the mitochondrial outer (x-axis) and inner membrane (y-axis). The closed symbols denoting R8 modified liposomes showed higher values compared with the open symbols denoting R8 unmodified liposomes in both $\mathrm{x}$ and $\mathrm{y}$ values, suggesting that R8-modification enhances the fusion activity of carriers for both mitochondrial outer and inner membranes. We also determined the relation between the mitochondrial outer and inner membrane fusion activities of a carrier by the weighted Pearson's correlation coefficient using the numbers of observations for each sample as the weight. A statically significant $(P<0.0001)$ correlation $(r=0.672)$ was observed. Collectively, these findings confirm that a conventional MITO-Porter containing SM and PA with a high fusion activity with respect to the mitochondrial outer membrane (Yamada et al., 2008) could also effectively fuse with the mitochondrial inner membrane.

\subsection{Evaluation of mitochondrial targeting and the matrix import of nucleic acids by MITO-Porter system using} isolated mitochondria.

We investigated mitochondrial targeting and the import of nucleic acids into the matrix using liposomes with various fusogenic properties. Liposomes encapsulating Cy-5 RNA oligo were incubated with isolated mitochondria, followed by centrifugation to remove the unbound liposomes, and the fluorescence intensity of Cy-5 RNA oligo in the mitochondrial fraction was measured to estimate mitochondrial targeting activity. In addition, a mitoplast suspension was prepared (outer membrane removed), followed by quantifying the Cy-5 RNA oligo to calculate the matrix import efficiency. Figure 3A shows the physicochemical properties (diameters, $\zeta$ potential, and encapsulation efficiency) and fusion activities of the constructed liposomes: R8-EPC/SM, R8-DOPE/PI and R8-DOPE/SM (MITOPorter (SM)).

In evaluating mitochondrial targeting activity (Figure 3B), all of the carriers showed efficient mitochondrial 
1 targeting of nucleic acids. This result suggests that the carriers are accessible to mitochondria regardless of their

2 mitochondrial membrane fusion activities, and is consistent with a previous report that R8-modified liposomes with various lipid compositions were able to bind to isolated mitochondria (Yamada and Harashima, 2008). Moreover, we confirmed that the carriers with a high outer membrane fusogenic activity (R8-DOPE/PI and R8-DOPE/SM) delivered nucleic acids to mitochondria more efficiently than R8-EPC/SM, which has a low outer membrane fusion activity, although the difference was not significant. It was presumed that outer membrane fusion might contribute to the carriers being retained on mitochondria after completion of the binding.

In evaluating matrix import efficiency (Figure 3C), the R8-DOPE/SM, which has a mitochondrial fusogenic lipid composition for the outer and inner membranes, showed a higher matrix import efficiency than the other carriers. R8-DOPE/PI with a low inner membrane activity showed a slightly lower matrix import efficiency compared with R8-DOPE/SM. On the other hand, R8-EPC/SM, with a low outer membrane activity, showed a significantly lower matrix import efficiency compared with R8-DOPE/SM. These results suggest that a carrier requires a fusion activity for the mitochondrial outer and inner membranes for nucleic acids to be delivered to the mitochondrial matrix. In particular, the outer membrane fusion process could largely contribute to mitochondrial matrix import as a first step. In our latest study, we showed that the MITO-Porter system containing DOPE, SM and STR-R8 resulted in the successful delivery of an antisense RNA oligonucleotide to mitochondria in living cells, and induced the knockdown of the targeted mitochondria-encoded mRNA and protein to regulate mitochondrial function (Furukawa et al., 2015). This result supports the findings showing that R8-DOPE/SM achieves the mitochondrial matrix delivery of nucleic acids via the mitochondrial outer and inner membrane fusion.

\section{Conclusion} mitochondrial outer and inner membranes, and that a combination of R8 and DOPE mainly contributed the inner membranes, similar to the outer membranes. Moreover, it was confirmed that the MITO-Porter delivered nucleic acids into the mitochondrial matrix, indicating that it holds promise as a mitochondrial vector for nucleic acids. Studies related to this issue are currently in progress. 


\section{Acknowledgements}

We thank Dr. Y. Shinohara (University of Tokushima, Tokushima, Japan) for kindly supplying primary antibodies from rabbit against VDAC2. This work was supported, in part by, a Grant-in-Aid for Young Scientists (A) [Grant No. 26282131 (to Y.Y.)], a Grant-in Aid for Challenging Exploratory Research [Grant No. 25560219 (to Y.Y.)] and a Grant-in-Aid for Scientific Research (B) [Grant No. 26282131 (to Y.Y.)] from the Ministry of Education,

6 Culture, Sports, Science and Technology, the Japanese Government (MEXT), and A-step feasibility study program in 7 Japan Science and Technology Agency (JST) [Grant No. AS251Z00277Q (to Y.Y.)], and a grant from Northern 8 Advancement Center for Science \& Technology (Noastec Foundation,. Hokkaido, Japan) [Grant No. T-1-42 (to $9 \quad$ Y.Y.)]. We also thank Dr. Milton Feather for his helpful advice in writing the manuscript. 


\section{References}

Biswas, S., Torchilin, V.P., 2014. Nanopreparations for organelle-specific delivery in cancer. Adv Drug Deliv Rev 66, 26-41, doi: 10.1016/j.addr.2013.11.004

Chan, D.C., 2006. Mitochondria: dynamic organelles in disease, aging, and development. Cell 125, 12411252, doi: 10.1016/j.cell.2006.06.010

Furukawa, R., Yamada, Y., Kawamura, E., Harashima, H., 2015. Mitochondrial delivery of antisense RNA by MITO-Porter results in mitochondrial RNA knockdown, and has a functional impact on mitochondria. Biomaterials 57, 107-115, doi: 10.1016/j.biomaterials.2015.04.022

Futaki, S., Ohashi, W., Suzuki, T., Niwa, M., Tanaka, S., Ueda, K., Harashima, H., Sugiura, Y., 2001. Stearylated arginine-rich peptides: a new class of transfection systems. Bioconjug Chem 12, 10051011, doi: 10.1021/bc0155081

Greenawalt, J.W., 1974. The isolation of outer and inner mitochondrial membranes. Methods Enzymol 31, 310-323, doi:

Kajimoto, K., Sato, Y., Nakamura, T., Yamada, Y., Harashima, H., 2014. Multifunctional envelope-type nano device for controlled intracellular trafficking and selective targeting in vivo. J Control Release 190C, 593-606, doi: 10.1016/j.jconrel.2014.03.058

Kawamura, E., Yamada, Y., Harashima, H., 2013. Mitochondrial targeting functional peptides as potential devices for the mitochondrial delivery of a DF-MITO-Porter. Mitochondrion 13, 610-614, doi: 10.1016/j.mito.2013.08.010

Khalil, I.A., Kogure, K., Futaki, S., Harashima, H., 2006. High density of octaarginine stimulates macropinocytosis leading to efficient intracellular trafficking for gene expression. J Biol Chem 281, 3544-3551, doi: 10.1074/jbc.M503202200

Kogure, K., Moriguchi, R., Sasaki, K., Ueno, M., Futaki, S., Harashima, H., 2004. Development of a nonviral multifunctional envelope-type nano device by a novel lipid film hydration method. J Control Release 98, 317-323, doi: 10.1016/j.jconrel.2004.04.024

S0168365904002159 [pii]

Maier, O., Oberle, V., Hoekstra, D., 2002. Fluorescent lipid probes: some properties and applications (a review). Chem Phys Lipids 116, 3-18, doi: 10.1016/S0009-3084(02)00017-8

Miyata, K., Nishiyama, N., Kataoka, K., 2012. Rational design of smart supramolecular assemblies for gene delivery: chemical challenges in the creation of artificial viruses. Chem Soc Rev 41, 2562-2574, doi: $10.1039 / \mathrm{c} 1 \mathrm{cs} 15258 \mathrm{k}$

Nakamura, T., Akita, H., Yamada, Y., Hatakeyama, H., Harashima, H., 2012. A multifunctional envelopetype nanodevice for use in nanomedicine: concept and applications. Accounts of chemical research 45, 1113-1121, doi: 10.1021/ar200254s

Schapira, A.H., 2006. Mitochondrial disease. Lancet 368, 70-82, doi: 10.1016/S0140-6736(06)68970-8

Struck, D.K., Hoekstra, D., Pagano, R.E., 1981. Use of resonance energy transfer to monitor membrane fusion. Biochemistry 20, 4093-4099, doi: 10.1021/bi00517a023

Taylor, R.W., Turnbull, D.M., 2005. Mitochondrial DNA mutations in human disease. Nat Rev Genet 6, 
389-402, doi: $10.1038 / \operatorname{nrg} 1606$

Weissig, V., 2011. From serendipity to mitochondria-targeted nanocarriers. Pharmaceutical research 28, 2657-2668, doi: 10.1007/s11095-011-0556-9

Yamada, Y., Akita, H., Harashima, H., 2012a. Multifunctional envelope-type nano device (MEND) for organelle targeting via a stepwise membrane fusion process. Methods Enzymol 509, 301-326, doi: 10.1016/B978-0-12-391858-1.00015-0

Yamada, Y., Akita, H., Kamiya, H., Kogure, K., Yamamoto, T., Shinohara, Y., Yamashita, K., Kobayashi, H., Kikuchi, H., Harashima, H., 2008. MITO-Porter: A liposome-based carrier system for delivery of macromolecules into mitochondria via membrane fusion. Biochimica et biophysica acta 1778, 423432, doi: 10.1016/j.bbamem.2007.11.002

Yamada, Y., Furukawa, R., Yasuzaki, Y., Harashima, H., 2011. Dual function MITO-Porter, a nano carrier integrating both efficient cytoplasmic delivery and mitochondrial macromolecule delivery. Mol Ther 19, 1449-1456, doi: 10.1038/mt.2011.99

Yamada, Y., Harashima, H., 2008. Mitochondrial drug delivery systems for macromolecule and their therapeutic application to mitochondrial diseases. Adv Drug Deliv Rev 60, 1439-1462, doi: 10.1016/j.addr.2008.04.016

Yamada, Y., Harashima, H., 2012. Delivery of bioactive molecules to the mitochondrial genome using a membrane-fusing, liposome-based carrier, DF-MITO-Porter. Biomaterials 33, 1589-1595, doi: 10.1016/j.biomaterials.2011.10.082

Yamada, Y., Harashima, H., 2014. A method for screening mitochondrial fusogenic envelopes for use in mitochondrial drug delivery. Methods in molecular biology 1141, 57-66, doi: 10.1007/978-1-49390363-4_2

Yamada, Y., Kawamura, E., Harashima, H., 2012e. Mitochondrial-targeted DNA delivery using a DFMITO-Porter, an innovative nano carrier with cytoplasmic and mitochondrial fusogenic envelopes. J Nanopart Res 14, 1013-1027, doi: Artn 1013

Doi 10.1007/S11051-012-1013-3

Yasuzaki, Y., Yamada, Y., Harashima, H., 2010. Mitochondrial matrix delivery using MITO-Porter, a liposome-based carrier that specifies fusion with mitochondrial membranes. Biochem Biophys Res Commun 397, 181-186, doi: 10.1016/j.bbrc.2010.05.070

Zhang, E., Zhang, C., Su, Y., Cheng, T., Shi, C., 2011. Newly developed strategies for multifunctional mitochondria-targeted agents in cancer therapy. Drug Discov Today 16, 140-146, doi: S13596446(10)00844-5 [pii]

10.1016/j.drudis.2010.12.006 


\section{$1 \quad$ Figure captions}

2 Fig. 1. Evaluation of the fusion activities of liposomes for mitochondrial outer membrane by FRET analysis using

3 isolated mitochondria. (A) Schematic image of membrane fusion activity of liposomes with mitochondria was assessed by FRET between donor and acceptor fluorophores, modified on the surface of liposomes. (B) Evaluation of fusion activities of various type-liposomes with respect to the mitochondrial outer membrane using isolated mitochondria and FRET analysis. Data are represented by the means \pm S.D. $(n=3-6) .{ }^{\# \#}$ Significant differences are calculated among "lipid composition" ( $P<0.001$ by two-way ANOVA, followed by Scheffe's test). There was significant interaction between two factors was detected $(P<0.01)$.

Fig. 2. Preparation of a mitoplast suspension and fusion activities of various type-liposomes for the mitochondrial inner membrane. (A) Schematic image to prepare mitoplast suspension. This procedure includes two steps; digitonin treatment of mitochondria to remove the outer membrane $(\mathrm{OM})$ and differential centrifugation to purify the mitoplast suspension, now comprised of the inner membrane and matrix. (B) Western blotting analysis to analyze purity of mitoplast suspension. Mitoplast was prepared using different concentration of digitonin $(0,0.3,0.6,0.9,1.2,1.5 \%)$. Each sample ( $2 \mu \mathrm{g}$ of protein) of mitoplast suspension was subjected to western blotting. Primary antibodies against the VDAC2 (a), locating OM and PDHE1- $\alpha$ (b), locating matrix were used. (C) Evaluation of fusion activities of various type-liposomes for mitochondrial inner membrane using mitoplasts and FRET analysis. Data are represented

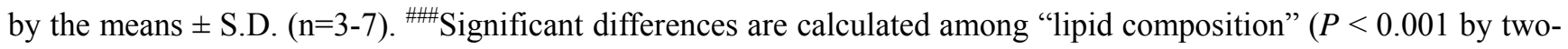
way ANOVA, followed by Scheffe's test). There was no interaction between two factors was detected $(P=0.63)$.

Fig. 3. Evaluation of mitochondrial targeting and the import of nucleic acids into the matrix using isolated mitochondria. Characteristics of the carriers encapsulating Cy5-RNA oligo for this evaluation are summarized in (A). For fusion activities, we used the data shown in Figure 1B and Figure 2C. Mitochondrial targeting activities (B) and mitochondrial import efficiencies $(\mathbf{C})$ of the carriers were evaluated using isolated rat liver mitochondria and the mitoplast, respectively. Data are means \pm S.D. $(n=3-4)$. Significant differences between unmodified liposome and peptide modified liposomes $\left({ }^{*} P<0.01,{ }^{*} P<0.05\right.$ by one-way ANOVA, followed by Student-Newman-Keuls (SNK) test). 
2 TABLE 1. Characteristics of liposomes

3

\begin{tabular}{|c|c|c|c|c|c|c|c|c|c|c|}
\hline \multirow{2}{*}{$\begin{array}{l}\text { Liposome } \\
\text { type }\end{array}$} & \multirow{2}{*}{ a Liposome composition } & \multicolumn{9}{|c|}{ Lipid X component and diameters $(D) / \xi$-potentials $(Z)$ of liposomes } \\
\hline & & & SM & Chol & CHEMS & PG & PA & CL & PI & PS \\
\hline \multirow{2}{*}{ EPC-LP } & EPC/lipid X & $D(\mathrm{~nm})$ & $110 \pm 14$ & $106 \pm 5$ & $107 \pm 16$ & $102 \pm 3$ & $99 \pm 3$ & $97 \pm 6$ & $97 \pm 4$ & $98 \pm 4$ \\
\hline & $(9: 2$, molar ratio $)$ & $Z(\mathrm{mV})$ & $-17 \pm 2$ & $-23 \pm 10$ & $-22 \pm 13$ & $-34 \pm 5$ & $-30 \pm 4$ & $-27 \pm 3$ & $-43 \pm 4$ & $-51 \pm 1$ \\
\hline \multirow{2}{*}{ DOPE-LP } & DOPE/lipid X & $D(\mathrm{~nm})$ & $122 \pm 33$ & $192 \pm 26$ & $235 \pm 51$ & $120 \pm 24$ & $146 \pm 31$ & $221 \pm 122$ & $147 \pm 6$ & $110 \pm 2$ \\
\hline & $(9: 2$, molar ratio $)$ & $Z(\mathrm{mV})$ & $-24 \pm 5$ & $-38 \pm 9$ & $-49 \pm 4$ & $-35 \pm 6$ & $-42 \pm 8$ & $-47 \pm 3$ & $-47 \pm 3$ & $-52 \pm 1$ \\
\hline \multirow{2}{*}{ R8-EPC-LP } & EPC/lipid X/STR-R8 & $D(\mathrm{~nm})$ & $106 \pm 5$ & $115 \pm 19$ & $110 \pm 20$ & $105 \pm 19$ & $107 \pm 25$ & $109 \pm 19$ & $96 \pm 3$ & $92 \pm 5$ \\
\hline & $(9: 2: 1$, molar ratio $)$ & $Z(\mathrm{mV})$ & $38 \pm 3$ & $42 \pm 6$ & $37 \pm 6$ & $38 \pm 8$ & $33 \pm 7$ & $38 \pm 3$ & $45 \pm 4$ & $42 \pm 1$ \\
\hline \multirow{2}{*}{ R8-DOPE-LP } & DOPE:lipid X:STR-R8 & $D(\mathrm{~nm})$ & $127 \pm 40$ & $208 \pm 28$ & $233 \pm 27$ & $130 \pm 7$ & $146 \pm 15$ & $186 \pm 47$ & $196 \pm 38$ & $122 \pm 5$ \\
\hline & $(9: 2: 1$, molar ratio) & $Z(\mathrm{mV})$ & $38 \pm 8$ & $50 \pm 5$ & $51 \pm 5$ & $35 \pm 6$ & $37 \pm 17$ & $47 \pm 11$ & $52 \pm 2$ & $46 \pm 1$ \\
\hline
\end{tabular}

$5{ }^{\text {a }}$ Lipid compositions in all of the prepared liposomes include both 1 mol\% NBD-DOPE and 0.5 mol\% rhodamine-

6 DOPE of the total lipids to investigate mitochondrial fusogenic activity using isolated mitochondria and FRET

7 analysis.

8 EPC, egg yolk phosphatidyl choline; DOPE, dioleoyl phosphatidyl ethanolamine; STR-R8, stearyl octaarginine; SM,

9 sphingomyelin; Chol, cholesterol; CHEMS, cholesteryl hemisuccinate; PG, phosphatidyl glycerol; PA, phosphatidic

10 acid; CL, cardiolipin; PI, phosphatidyl inositol; PS, phosphatidyl serine. Each value is represented by the mean \pm SD

$11 \quad(\mathrm{n}=4-6)$ 


\begin{tabular}{|c|c|c|c|c|c|c|}
\hline \multirow{2}{*}{ Carriers type } & \multirow{2}{*}{ Lipid composition (molar ratio) } & \multirow{2}{*}{$\begin{array}{l}\text { Diameter } \\
(\mathrm{nm})\end{array}$} & \multirow{2}{*}{$\begin{array}{l}\zeta \text { potential } \\
(\mathrm{mV})\end{array}$} & \multirow{2}{*}{$\begin{array}{l}\text { Encapsulation } \\
\text { efficiency }(\%)\end{array}$} & \multicolumn{2}{|c|}{ Fusion activity $(\%$} \\
\hline & & & & & OM & IM \\
\hline R8-EPC/SM & EPC/SM/CHEMS/STR-R8 (9:2:1:1) & $67 \pm 5$ & $28 \pm 1$ & $58 \pm 3$ & 25 & 46 \\
\hline R8-DOPE/PI & DOPE/PI/CHEMS/STR-R8 (9:2:1:1) & $132 \pm 18$ & $49 \pm 2$ & $49 \pm 2$ & 60 & 34 \\
\hline R8-DOPE/SM & DOPE/SM/CHEMS/STR-R8 (9:2:1:1) & $162 \pm 17$ & $43 \pm 1$ & $76 \pm 3$ & 51 & 52 \\
\hline
\end{tabular}

B

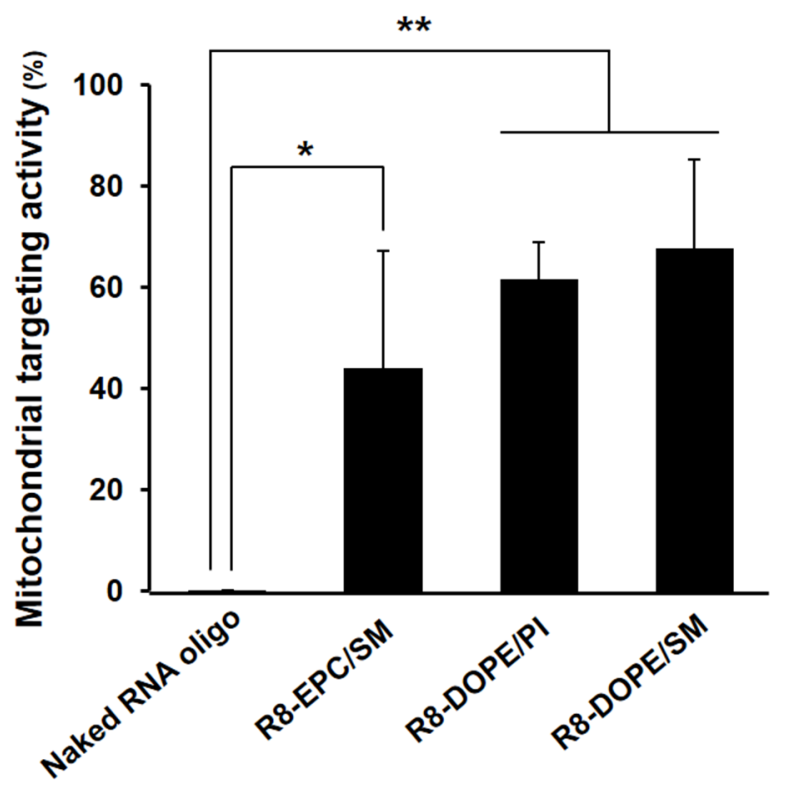

C

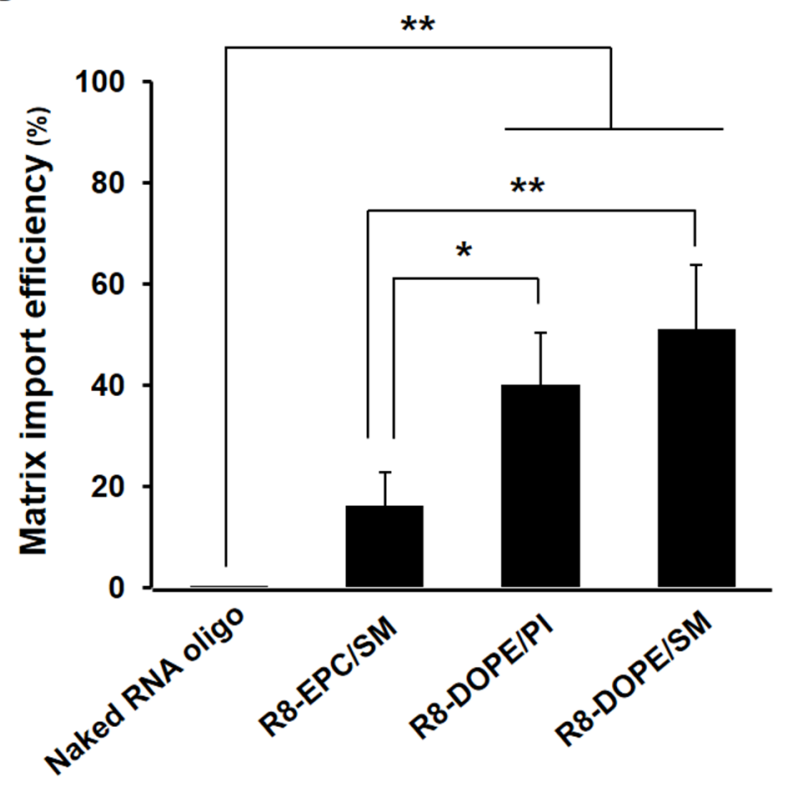

